\title{
Anomalous Beam Effects during in situ Transmission Electron Microscopy Deformation of Nanocrystalline and Ultrafine-grained Metals
}

\author{
Rohit Sarkar $^{1}$, Christian Rentenberger ${ }^{2}$, Jagannathan Rajagopalan ${ }^{1}$ \\ 1. School for Engineering of Matter Transport and Energy, Arizona State University, Tempe 85287, \\ USA. \\ 2. Physics of Nanostructured Materials, Faculty of Physics, University of Vienna, Boltzmanngasse 5, \\ 1090 Vienna, Austria.
}

It is well established that the TEM e-beam can cause radiation damage to specimen. Radiolysis, knockon damage and specimen heating are known to be the most common damage mechanisms [1]. Apart from these conventional damage mechanisms, the e-beam has also been recently shown to alter the deformation mechanisms in nanomaterials. The e-beam can activate dislocations, and defects generated by e-beam exposure can lead to additional deformation processes. For example, low to moderate intensity e-beam has been used to induce superplastic deformation in nanoscale silica particles and nanowires that are usually brittle at low temperatures [2].

Since nanocrystalline and ultrafine-grained metals have a high density of crystal defects (nonequilibrium grain boundaries and associated extrinsic dislocations) that can be activated by the e-beam, it is quite conceivable that e-beam exposure will alter the mechanical behavior of such materials during in situ experiments. However, the e-beam effect on the in situ deformation response of these materials remains unknown.

In the following paper we provide direct evidence that e-beam exposure of nanocrystalline and ultrafinegrained aluminum and gold films causes increased defect activity leading to substantial changes in its stress-strain response. We systematically investigated the effects of e-beam during in-situ load-unload experiments, while simultaneously measuring the macroscopic stress-strain response.

MEMS based tensile testing stages were co-fabricated with freestanding Al and Au thin films, deposited by DC magnetron sputtering, using microfabrication techniques. A GATAN TM TEM straining holder was then used to deform these MEMS devices containing the films in a Phillips CM200 TEM. In the first set of experiments, the stress-strain response of the films was measured over multiple cycles. During the first cycle the films were deformed without e-beam exposure to serve as a reference. In the subsequent cycles, the films were exposed to the e-beam at different accelerating voltages and the area of the film exposed to the beam as well as the beam intensity were systematically varied. In the second set of experiments the film was exposed to the e-beam only at two points during each loading cycle to quantify e-beam induced stress relaxation.

We found that e-beam exposure caused both significant stress relaxation and anomalous changes in sample geometry in nanocrystalline and ultrafine-grained aluminum and gold films [3]. Beam-induced artifacts increased with e-beam intensity but, contrary to expectation, the artifacts were more pronounced at lower accelerating voltages. Furthermore, e-beam exposure induced anomalous sample necking, which unusually depended on the beam diameter rather than intensity. These observations in two metals with highly dissimilar atomic weights and properties indicate that a broad spectrum of nanostructured materials are susceptible to beam-induced artifacts, and simultaneously point to a strategy to minimize such artifacts [4]. 


\section{References:}

[1] D.B. Williams, C.B. Carter, The Transmission Electron Microscope, in: Transmission Electron Microscopy, Springer US, 2009: pp. 3-22. http://link.springer.com/chapter/10.1007/978-0-387-765013 1 (accessed March 25, 2015).

[2] K. Zheng et al, Nat Commun. 1 (2010), p. 24. doi:10.1038/ncomms1021.

[3] R. Sarkar, C. Rentenberger, J. Rajagopalan, Sci Rep. 5 (2015). doi:10.1038/srep16345.

[4] This project was funded by the National Science Foundation (NSF) grants ECCS 1102201, CMMI 1400505 and DMR 1454109. The authors would like to gratefully acknowledge the use of facilities at the John M. Cowley Centre for High Resolution Electron Microscopy and the Centre for Solid State Electronics Research at Arizona State University and at the Faculty of Physics of the University of Vienna. Financial support from the Austrian Science Fund (FWF): [P22440, I1309] is acknowledged.
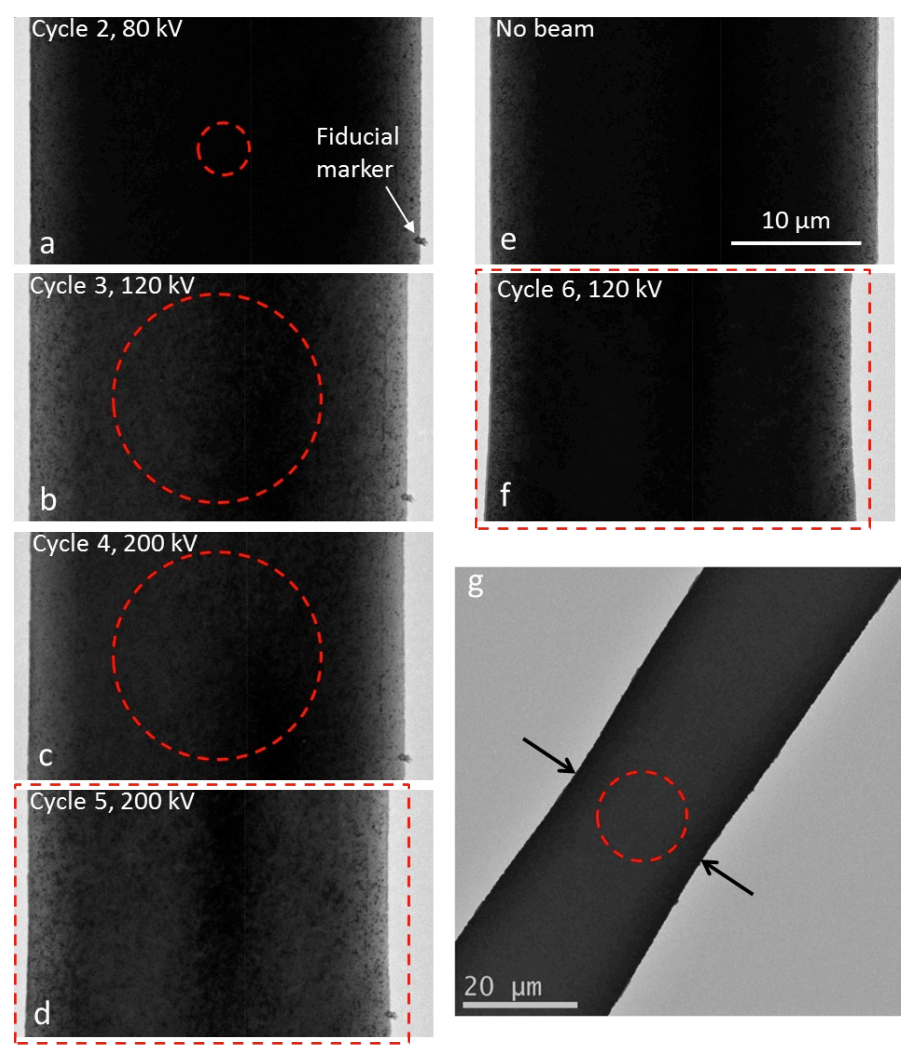

Figure 1. The e-beam caused anomalous necking along the sample width. Necking was more pronounced at $120 \mathrm{kV}$ and depended more on area of specimen exposed to beam (red circle/square) than intensity. 